\title{
LEARNING-INDUCED MODULATION OF SCALE-FREE PROPERTIES OF BRAIN ACTIVITY MEASURED WITH MEG
}

\author{
Nicolas Zilber ${ }^{(1,2)}$, Philippe Ciuciu ${ }^{(1,2)}$, Patrice Abry ${ }^{(3)}$ and Virginie van Wassenhove ${ }^{(1,4,5)}$ \\ ${ }^{(1)}$ CEA/DSV/I²BM NeuroSpin center, Bât. 145, F-91191 Gif-sur-Yvette, France \\ (2) Parietal Project Team, INRIA Saclay Ile-de-France, 91893 Orsay, France. \\ (3) CNRS, UMR 5672, Laboratoire de Physique. ENS Lyon, France. \\ (4) INSERM, U992, Cognitive Neuroimaging Unit, F-91191 Gif-sur-Yvette, France. \\ (5) Univ Paris-Sud, Cognitive Neuroimaging Unit, F-91191 Gif-sur-Yvette, France. \\ 1 firstname. lastnamedcea.fr, ${ }^{3}$ patrice.abrydens-lyon. fr
}

\begin{abstract}
Previous studies have suggested that infraslow brain activity could play an important role in cognition. Its scale-free properties (coarsely described by its $1 / f$ power spectrum) are indeed modulated between contrasted conscious states (sleep vs. awake). However, finer modulations remain to be investigated. Here, we make use of a robust multifractal analysis to investigate the group-level impact of perceptual learning (visual (V), or audiovisual (AV), $N=12$ subjects in each group) on the source reconstructed scale-free activity recorded with magnetoencephalography (MEG) during rest and task. We first observed a significant decrease of self-similarity in evoked activity during the task after both trainings. More interestingly, only the most efficient training (AV) induced a decrease of self-similarity in spontaneous activity at rest whereas only $\mathrm{V}$ training induced an increase of multifractality in evoked activity.
\end{abstract}

Index Terms - MEG, scale-free activity, scaling, multifractal analysis, plasticity, learning

\section{INTRODUCTION}

Traditionally, the analysis of brain signals acquired with electroencephalography (EEG) and magnetoencephalography (MEG) has consisted of decomposing the temporal signal into different oscillations whose prominent frequency peaks were easily observable in the power spectrum. These frequency bands were given names suchs as $\delta(1-3 \mathrm{~Hz}), \theta(4-8 \mathrm{~Hz}), \alpha(8-12 \mathrm{~Hz}), \beta(12-30 \mathrm{~Hz})$ and $\gamma(>40 \mathrm{~Hz})$. They drew all the attention to the detriment of infraslow activity $(<1 \mathrm{~Hz})$, which is characterized by a power law distribution. In other words, its power spectrum plotted in logarithmic coordinates decreases linearly. Because such behavior (also referred to as $1 / f$ spectrum) is very common in dynamical systems (e.g. turbulence, stock market...) [1], this infraslow activity has long been attributed to sensor or neural noise and considered to be functionally irrelevant. However, since the last decade, a growing body of evidence has shown a modulation of the $1 / f$ slope between contrasted cognitive states (awake vs phases of sleep [1,2], task vs rest [3,4]) and in pathologies [5] using different imaging techniques (ECoG [1,6], EEG [7-9], MEG [8, 10] and $\mathrm{fMRI}[3,6,11,12])$. Such results suggest that the infraslow activity also carries meaningful information for brain function.

A $1 / \mathrm{f}$ spectrum can be interpreted in time domain as a scalefree property. A signal $X(t)$ with such properties is said to be selfsimilar, meaning that it shares the same statistical properties with its own dilated version $X(a t)_{a>0}$ scaled by the factor $a^{-H}$. The exponent $H$ is called the self-similarity parameter or Hurst exponent. If the signal can be modelled as a fractional Gaussian noise (fGn) or its increments as a fractional Brownian motion ( $\mathrm{fBm}$ ), the Hurst exponent $H$ is linearly related to the $1 / f$ slope and can be accurately estimated using the Detrended Fluctuation Analysis (DFA) [13] or even more simply with a linear fit on the log-scale power spectrum. However, experimental data in different neuroimaging modalities often presents non-Gaussian and non-stationnary properties, thus significantly altering the reliability and statistical perfomances of such analysis tools. Instead, another analysis, more robust to non-stationarity issues and more efficient in disentangling true scaling phenomena from superimposed drifts has been proposed since the last decade as the Wavelet Leader Based Multifractal Formalism (WLBMF) [14]. This method offers a richer and more flexible description of functional neuroimaging data and has been successfully applied to MEG in previous work [10] to demonstrate a modulation of scale-free properties of MEG time series at the sensor level between evoked and spontaneous activity.

According to recent findings showing that spontaneous activity at rest is modified by learning [15], we now asked whether refined modulations of scale-free properties could be observed after visual learning in ongoing and evoked activity. To this aim, two groups of participants were trained to discriminate the coherence level of two mixed random dot kinematograms (RDK). The training was adjusted individually and performed either audiovisually (AV) or visually $(\mathrm{V})$ only. We then carried out the WLBMF analysis on MEG data reconstructed onto cortical areas presenting functional plasticity.

Here, we propose an intuitive interpretation of the two main measures output by WLBMF, which are self-similarity and multifractality (see Section 2). We also show that the AV training is more efficient than the $\mathrm{V}$ training and we present the cortical areas selected for the WLBMF analysis (see Section 3). Finally, differences in self-similarity and multifractality before and after learning in spontaneous and evoked activity for both trainings are presented (see Section 4). A significant decrease of self-similarity is reported in $i$.) evoked activity after both trainings ( $\mathrm{V}$ and $\mathrm{AV}$ ), and in ii.) spontaneous activity after AV training only. On the other hand, a significant increase of multifractality could be exhibited only during evoked activity after V training. Conclusions are drawn in Section 5.

\section{SCALING ANALYSIS: INTUITIVE APPROACH}

\subsection{Self-similarity}

As previously mentioned, a self-similar process has the same statistical properties as its rescaled dilated version, which means that their 
statistical moments are equal at any order. Contrary to standard scaling analysis which only examines the second statistical order using a power spectrum estimation, the WLBMF method accounts for all orders. This provides a very rich description of the scaling properties, often given in the form of the multifractal spectrum. This spectrum is in practice often reduced to its truncated $2^{\text {nd }}$ order polynomial expansion with only two parameters: self-similarity and multifractality. A complete and more formal description has been given in previous articles [4,14] (where self-similarity is measured by attribute $c_{1}^{L}$ and multifractality by attribute $c_{2}^{L}$ ) and will not be recalled hereafter.

Self-similarity can be approximated here by the $1 / f$ slope and more precisely by the Hurst exponent. These values are exactly the same in the absence of multifractality, e.g. for fGn or fBm processes. This measure also reflects the long memory of the process: if its selfsimilarity is strong, its autocorrelation decays very slowly with the lag. In other words, what happens now will still have some influence long time after. In the same vein, self-similarity also reflects the global regularity of the signal: as it increases, the signal becomes smoother. These properties converge to the following neurophysiological interpretation, already suggested in [3,5]: A decrease of selfsimilarity indicates an increase of neural excitability.

Such interpretation is consistent with the experimental result illustrated in Fig. 1, showing the Welch's power spectrum estimates in log-log scale of MEG data in one sensor acquired during the execution of a task (red), resting state (green) and in empty room (light blue): the $1 / f$ slope at rest is steeper than during task. This observation is consistent with other studies that have found stronger self-similarity (i.e. weaker neural excitability) during rest than during visual or auditory detection tasks $[3,4]$.

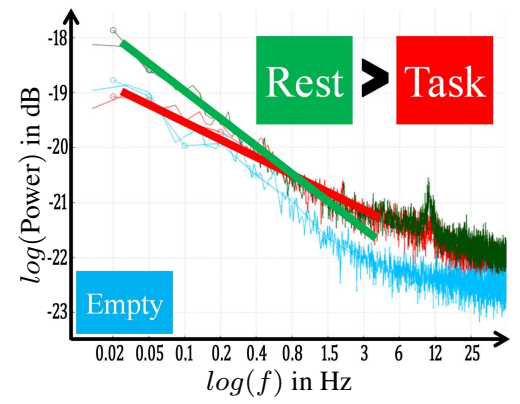

Fig. 1. Modulation of the 1/f slope between rest and task. Welch's periodograms of MEG data are plotted in red (task), green (rest) and light blue (empty). The $1 / f$ slope at rest is steeper than during task.

\subsection{Multifractality}

In the absence of multifractality, the scaling properties are entirely described by only one parameter: self-similarity. In this case, the scaling behavior is the same for all statistical orders; it would thus be enough to examine only the $1 / f$ slope at the $2^{\text {nd }}$ statistical order. This assertion is no longer valid in presence of multifractality.

A more intuitive approach illustrated in Fig. 2 is to consider multifractality as local fluctuations over time around the $1 / f$ slope. Imagine you can estimate the power spectrum at each time point perfectly. The $1 / f$ slope would fluctuate around a mean value corresponding to the Hurst exponent or self-similarity captured by parameter $c_{1}^{L}$. Interestingly, these fluctuations are not random but present a structured pattern over time. Multifractality actually depicts their variance which is reflected by the second parameter $c_{2}^{L}$. To the best of our knowledge, no neurophysiological interpretations have yet been proposed as regards multifractality.

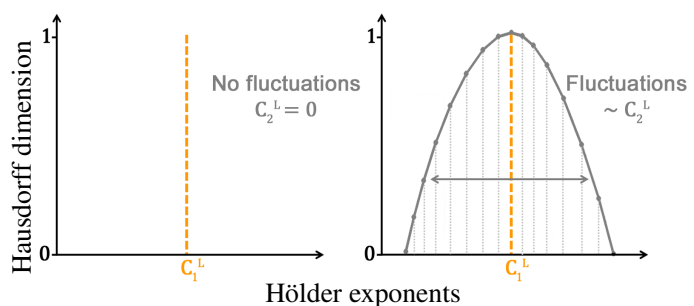

Fig. 2. Multifractal spectra with or without local fluctuations. In the absence of multifractality (left), the multifractal spectrum shows a single Hölder exponent also named Hurst exponent $\left(c_{1}^{L}\right)$. Local fluctuations over time around this exponent changes the spectrum into a concave curve ( right) whose broadness corresponds to the amount of multifractality $\left(c_{2}^{L}\right)$.

\section{LEARNING PARADIGM}

\subsection{Data acquisition}

Twenty-four healthy right-handed subjects (aged 19-27 years) with normal or corrected-to-normal vision participated in this experiment. Each participant provided an informed consent in accordance with the Declaration of Helsinki (2008) and the Ethics Committee on $\mathrm{Hu}-$ man Research at NeuroSpin (Gif-sur-Yvette, France).

Brain magnetic fields were recorded in a magnetically shielded room using the whole-head Elekta Neuromag MEG system (Elekta LTD, Helsinki) with 102 triple-sensor elements in upright position. Data were sampled at $2000 \mathrm{~Hz}$ and pass-band filtered between $0.03 \mathrm{~Hz}$ and $600 \mathrm{~Hz}$. Signal Space Separation (SSS) was carried out with MaxFilter (Elekta LTD) to remove external interferences. Occular and cardiac artifacts were removed using principal component analysis based on the recorded electrooculograms (EOG) and electrocardiograms (ECG).

Individual forward solutions for cortical source locations were computed using a 3-layers boundary element model (BEM) constrained by the individual's anatomical MRI. The inverse solution was computed using a weighted minimum norm estimate (MNE) [16]. For each region of interest, signals reconstructed in each of its vertices were averaged together and the resulting signal was submitted to the WLBMF analysis.

\subsection{Paradigm}

Before each experiment, empty room MEG recordings were acquired for $5 \mathrm{~min}$. The experiment began with $5 \mathrm{~min}$. of resting state recording (eyes open, fixating a black screen) followed by a visual test of approximately $12 \mathrm{~min}$. in which the perceptual threshold was assessed.

The task consisted of discriminating among two mixed colored RDKs (red and green) which one of them was more coherent during $1 \mathrm{~s}$. The motion coherence is defined by the proportion of dots moving in the same direction (radius of the annulus: 4-15 deg, dot radius: $0.2 \mathrm{deg}$, dot flow: 16.7 dots per $\mathrm{deg}^{2} . \mathrm{s}$, dot speed: $10 \mathrm{deg} / \mathrm{s}$ ). Subjects received next training adapted to their initial performance for approximately $40 \mathrm{~min}$. including $20 \mathrm{~min}$. of rest. Half of them received only visual $(\mathrm{V})$ training, whereas the other half benefited from audiovisual (AV) training during which acoustic textures correlated with the coherent RDK were simultaneously played. After the training, a new block of resting state was recorded as well as another visual test to assess the participant's improvement.

\subsection{Behavioral results}

We compared the progression of the perceptual threshold, defined as the motion coherence corresponding to $75 \%$ of performance, as a 
function of the training ( $\mathrm{V}$ or $\mathrm{AV}$ ). Results are shown with boxplots in Fig. 3. A decrease of threshold corresponds to an improvement and indicates therefore a learning effect.

We first checked the success of each training separately with a one-tailed paired $t$-test comparing the threshold before and after learning. Each training shows a significant decrease of threshold (V: $t_{11}=5.3, p<10^{-3}, \mathrm{AV}: t_{11}=9.4, p<10^{-6}$ ). We also compared the initial and final thresholds between the two trainings with a two-tailed two-samples $t$-test. Whereas $\mathrm{V}$ and $\mathrm{AV}$ groups are not significantly different initially $\left(t_{22}=0.29, p=0.77\right)$, the $\mathrm{AV}$ group performs significantly better than the $\mathrm{V}$ group after training $\left(t_{22}=3.1, p<10^{-2}\right)$. These differences are only due to participants' training history.

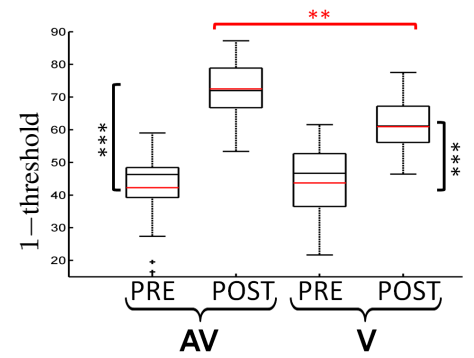

Fig. 3. Performance before and after training. Box plots show the distribution of perceptual thresholds in the AV (left) and V (right) groups in the pre- and post-training sessions. The median is reported in black and the average in red. Boxes contain values between the first and the third quartiles. Whiskers extend to the 5th and 95th percentiles. $* *$ and $* * *$ indicate a p-value inferior to $10^{-2}$ and $10^{-3}$, respectively.

\subsection{Regions of interest}

An event-related fields (ERF) analysis made in parallel (results not shown) allowed us to exhibit five cortical areas on the left hemisphere that presented significant differences of amplitude after $\mathrm{V}$ or AV training: 1.) the hMT+/V5 complex involved in visual motion processing, 2.) the visual area $\mathrm{V} 4$ involved in color processing, 3.) the inferotemporal cortex (ITC) involved in object recognition, 4.) the middle superior temporal sulcus (mSTS) involved in multisensory processing and 5.) the posterior superior temporal sulcus (pSTS) also involved in motion and multisensory processing. Fig. 4 shows these regions of interest (ROIs) averaged across all subjects on the template cortical surface of the left hemisphere.

Crucially, specific functional plasticity in hMT+/V5 was only induced by the AV learning, confirming the effectiveness of the AV training.

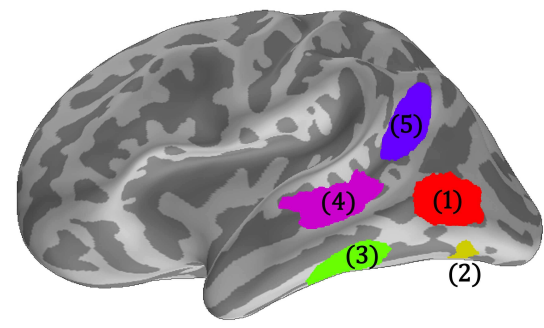

Fig. 4. Regions of interest. The five ROIs, revealed by our ERF analysis:1.) hMT+/V5, 2.) V4, 3.) ITC, 4.) mSTS and 5.) pSTS.

\section{MODULATION OF MULTIFRACTAL PROPERTIES WITH LEARNING}

\subsection{Difference in self-similarity}

For each ROI and for each subject, we estimated the self-similarity (denoted by $c_{1}^{L}$ ) of the reconstructed MEG signal during the rest and the execution of the task before and after training. The WLBMF analysis was carried out on the integrated time series (i.e. the cumulative sum) over the scale range $j=[10,14]$ (i.e. over the frequency range $f=[0.1,1.5] \mathrm{Hz}$ ) using a Daubechies mother wavelet with $N=3$ vanishing moments.

The mean differences $\Delta \widehat{c_{1}^{L}}=\widehat{c_{1}^{L}}$ POST $-\widehat{c_{1}^{L}}$ PRE after AV (left) and $\mathrm{V}$ (right) training in each ROI are shown in Fig. 5. A one-tailed one-sample $t$-test was carried out in each ROI to assess the statistical significance of the difference. Non-significant differences $(p \geqslant$ $0.05)$ are indicated with grey colors and group-level standard deviations are indicated with bars. Significant decreases of self-similarity (i.e. increases of neural excitability) are reported after both trainings in hMT+/V5, V4 and pSTS. Although only AV training yields a significant decrease of self-similarity in ITC and mSTS, the scale-free properties of the evoked activity evolved globally in the same direction irrespective of the training type.

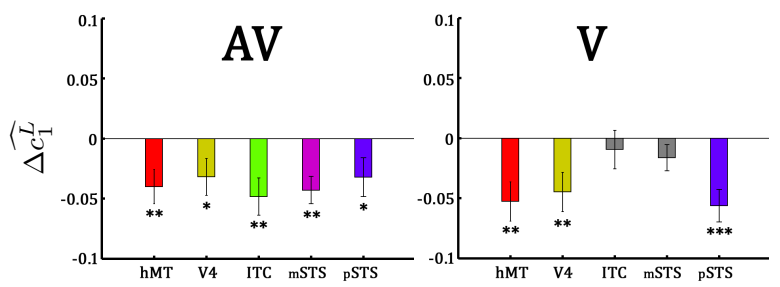

Fig. 5. Differences of self-similarity before and after training during task. ROIs presenting no significant differences are colored in grey. $*, * *$ and $* * *$ indicate a p-value inferior to $0.05,0.01$ and 0.001 , respectively

However, if we look at the mean differences of self-similarity in brain signals recorded at rest before and after training, we observe significant decreases only for the AV group in hMT+/V5, V4 and ITC, as shown in Fig. 6. In contrast to evoked activity, the training type is better distinguished in the resting state activity (i.e. ongoing fluctuations) in which only AV trained participants present an increase of neural excitability in cortical areas involved in plasticity.

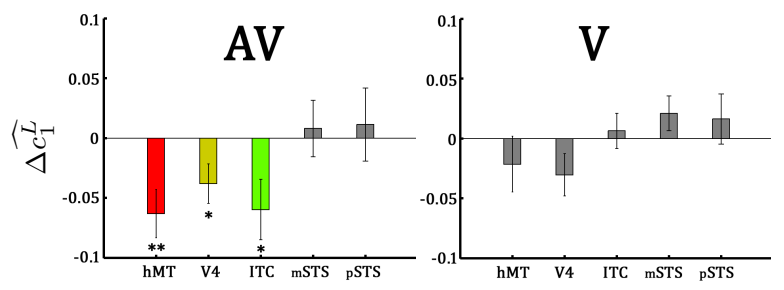

Fig. 6. Differences of self-similarity before and after training at rest. ROIs presenting no significant differences are colored in grey. $*$ and $* *$ indicate a p-value inferior to 0.05 and 0.01 , respectively.

\subsection{Difference in multifractality}

Multifractality (denoted by parameter $c_{2}^{L}$ ) was jointly estimated with self-similarity during the WLBMF analysis. Parameter $c_{2}^{L}$ is by construction negative, and thus the more negative $c_{2}^{L}$, the more signifi- 
cant multifractality. For ease of exposition, multifractality is discussed here in terms of $\left|c_{2}^{L}\right|$. Therefore, a positive mean difference $\Delta\left|\widehat{c_{2}^{L}}\right|=\left|\widehat{c_{2}^{L}}\right|_{\mathrm{POST}}-\left|\widehat{c_{2}^{L}}\right|_{\mathrm{PRE}}$ corresponds to an increase of multifractality.

Fig. 7 shows the mean differences of multifractality $\Delta\left|\widehat{c_{2}^{L}}\right|$ between pre- and post-training in evoked activity. Again, a one-tailed one-sample $t$-test was carried out in each ROI to assess the statistical significance of the difference. We only found significant increases of multifractality in the V group in hMT+/V5, V4 and mSTS, suggesting a modulation of the multifractal properties during evoked activity with the training type. Moreover, no significant mean differences of multifractality at rest could be found, as reported in Fig. 8.

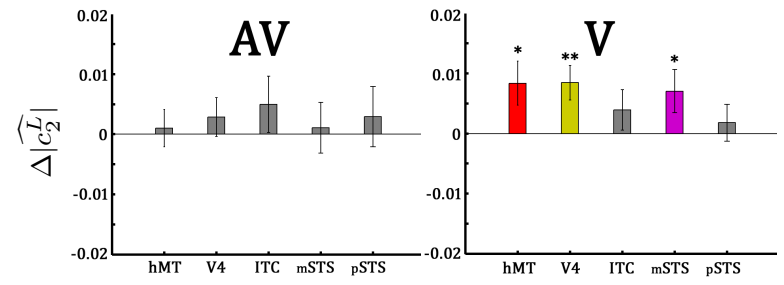

Fig. 7. Differences of multifractality before and after training during task. ROIs presenting no significant differences are colored in grey. $*$ and $* *$ indicate a p-value inferior to 0.05 and 0.01 , respectively.

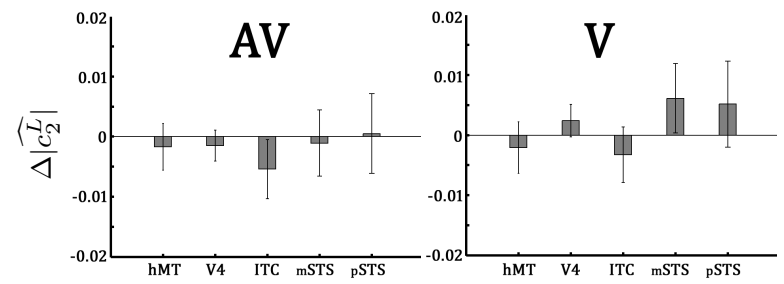

Fig. 8. Differences of multifractality before and after training at rest As no significant differences were found, all ROIs are colored in grey.

\section{DISCUSSION AND CONCLUSION}

In this study, we analyzed for the first time the scale-free properties of brain signals in MEG reconstructed on the cortical surface. In this manner, we could identify the neural sources for each individual and overcome the classic issue of normalization accross the participants that arises in sensor space.

Our results show a reduction of self-similarity in evoked activity that occurs after both trainings, without clear distinction between the natures of the learning process. We interpret it as an increase of the neural excitability which allows the participants to respond more quickly after the stimulus onset. Indeed, all participants' reaction time after training considerably decreased irrespective of the training type. These changes can also be attributed to a highest level of attentional focus or confidence.

Moreover, we observed an additional reduction of self-similarity in spontaneous activity after AV training only. Given that AV training was not only the most effective training but also the only one to induce specific plasticity in hMT+/V5, we suggest that these changes in self-similarity of resting-state activity capture functional plasticity. This is consistent with other studies in fMRI showing that learning sculpts resting-state activity [15].

No explanation has been given yet concerning the sporadic presence of multifractality in brain signals. Here, we observed an increase of multifractality during the task only after V training. These fluctuations of scale-free properties might reflect transient changes induced by an unachieved training that would disappear as soon as the asymptot is reached (i.e. like in AV learning). They might also reflect an attentional modulation that could indicate the absence of a true perceptual learning. Future work will be devoted to disentangle these two hypotheses.

\section{REFERENCES}

[1] B.J He, J.M Zempel, A.Z Snyder, and M.E Raichle, "The Temporal Structures and Functional Significance of Scale-free Brain Activity," Neuron, vol. 66, no. 3, pp. 353-369, May 2010.

[2] B Weiss, Z Clemens, R Bódizs, Z Vágó, and P Halász, "Spatiotemporal analysis of monofractal and multifractal properties of the human sleep EEG.," J Neurosci Methods, vol. 185, no. 1, pp. 116-124, Dec. 2009.

[3] B.J He, "Scale-Free Properties of the Functional Magnetic Resonance Imaging Signal during Rest and Task," J Neurosci, vol. 31, no. 39, pp. 13786-13795, Sep. 2011.

[4] P Ciuciu, G Varoquaux, P Abry, S Sadaghiani, and A Kleinschmidt, "Scale-Free and Multifractal Time Dynamics of fMRI Signals during Rest and Task.," Front Physiol, vol. 3, no. 6, pp. 186, Jan. 2012.

[5] V Maxim, L Sendur, J Fadili, J Suckling, R Gould, R Howard, and E Bullmore, "Fractional Gaussian noise, functional MRI and Alzheimer's disease.," Neuroimage, vol. 25, no. 1, pp. 141-158, Mar. 2005.

[6] J.M Palva and S Palva, "Infra-slow fluctuations in electrophysiological recordings, blood-oxygenation-level-dependent signals, and psychophysical time series.," Neuroimage, vol. 62, no. 4, pp. 2201-2211, Feb. 2012.

[7] D Van de Ville, J Britz, and C.M Michel, "EEG microstate sequences in healthy humans at rest reveal scale-free dynamics.," Proc Natl Acad Sci USA, vol. 107, no. 42, pp. 18179-18184, Oct. 2010.

[8] N Dehghani, C Bédard, S.S Cash, E Halgren, and A Destexhe, "Comparative power spectral analysis of simultaneous elecroencephalographic and magnetoencephalographic recordings in humans suggests non-resistive extracellular media.," J Comput Neurosci, vol. 29, no. 3, pp. 405-421, Dec. 2010.

[9] D Popivanov, V Stomonyakov, Z Minchev, S Jivkova, P Dojnov, S Jivkov, E Christova, and S Kosev, "Multifractality of decomposed EEG during imaginary and real visual-motor tracking.," Biol Cybern, vol. 94, no. 2, pp. 149-156, Feb. 2006.

[10] N Zilber, P Ciuciu, P Abry, and V van Wassenhove, "Modulation of scale-free properties of brain activity in MEG," in Proc IEEE ISBI, Barcelona, Spain, 2012, number 1, pp. 1531-1534.

[11] P Ciuciu, G Varoquaux, P Abry, and M Almog, "Multifractal analysis of resting state networks in functional MRI," in Proc IEEE ISBI, Chicago, 2011, number 1, pp. 473-478, IEEE.

[12] A.M Wink, E Bullmore, A Barnes, F Bernard, and J Suckling, "Monofractal and multifractal dynamics of low frequency endogenous brain oscillations in functional MRI.," Hum Brain Mapp, vol. 29, no. 7, pp. 791-801, July 2008.

[13] K Linkenkaer-Hansen, V.V Nikouline, J.M Palva, and R.J Ilmoniemi, "Long-range temporal correlations and scaling behavior in human brain oscillations.," J Neurosci, vol. 21, no. 4, pp. 1370-1377, Feb. 2001.

[14] H Wendt, P Abry, and S Jaffard, "Bootstrap for Empirical Multifractal Analysis," IEEE Signal Process Mag, vol. 24, no. 4, pp. 38-48, July 2007.

[15] C.M Lewis, A Baldassarre, G Committeri, G.L Romani, and M Corbetta, "Learning sculpts the spontaneous activity of the resting human brain.," Proc Natl Acad Sci USA, vol. 106, no. 41, pp. 17558-17563, Oct. 2009.

[16] M.S Hämäläinen and R.J Ilmoniemi, "Interpreting magnetic fields of the brain: minimum norm estimates," Med Biol Eng Comput, vol. 32, no. 1, pp. 35-42, 1994. 